\title{
Revisão sistemática de estudos qualitativos brasileiros com pessoas diagnosticadas como deficientes intelectuais
}

\author{
Fabiola Ribeiro de Souza \\ Silviane Bonaccorsi Barbato
}

\section{RESUMO}

Foram revisadas metodologias qualitativas em pesquisas brasileiras com indivíduos Deficientes Intelectuais (DI) publicadas nos últimos 16 anos, a fim de propor avanços metodológicos sobre produção de si em narrativas, a partir de críticas de pesquisadores culturais. As buscas foram feitas no Portal de Periódicos da CAPES, Google acadêmico e contato com pesquisadores, resultando em 20 estudos. Os resultados indicaram metodologias inovadoras e informam sobre condições de socialização inclusiva, na contemporaneidade brasileira. Para avançar no estudo de metodologias, sugerimos pesquisas em Teoria Fundamentada, a participação de pessoas com DI na análise e estudos sobre emoções.

Palavras-chaves: Revisão literatura, metodologia, deficiência intelectual, psicologia, cultura.

\section{ABSTRACT \\ Systematic review on brazilian qualitative studies with people diagnosed with intellectual disability}

16 years of Brazilian qualitative methodologies with people with Intellectual Disability (ID) were reviewed as a mean to propose methodological advances about self production in narratives, taking into consideration criticism made by cultural researchers. The search was done in the main Brazilian academic index, Google Scholar and academic contacts, resulting in 20 studies. The results show innovative methodologies and inform on inclusive socialization conditions in Brazilian contemporaneity. To further the study of methodologies, we suggest Grounded Theory studies, as well as the inclusion of ID people in the analyses and studies of emotions.

Keywords: Literature review, intellectual disability, methodology, culture, psychology.

Neste trabalho, revisamos a literatura de estudos brasileiros que utilizam métodos qualitativos (Atkinson, 1988; Bruner, 1997; McAdams \& Guo, 2015) com indivíduos Deficientes Intelectuais (DI), realizados nos últimos 16 anos. 0 objetivo foi verificar como estas investigações vêm sendo conduzidas nesta cultura, a fim de propor avanços metodológicos sobre produção de interpretação de si em narrativas, considerando-se ressalvas feitas por pesquisadores culturalistas (Brockmeier, 2012; Chakkarat, 2011; Mignolo, 2009; Straub, 2010, 2013) sobre o conhecimento científico, metodologias e interpretações de dados.

A escolha por estudos nacionais foi feita tendo em vista um dos pressupostos da psicologia cultural na vertente Indigenous (Chakkarat, 2011) em que muitos estudos, por questões epistemológicas e históricas, tendem a adotar metodologias ou interpretar fenômenos e experiências desconsiderando diferentes práticas culturais. Isto dificulta a compreensão do desenvolvimento humano na diversidade de condições de socialização, uma vez que alguns fenômenos podem ser comuns a certas culturas ou se concretizem de diferentes formas.

Mignolo (2009) chama atenção para a existência de uma "geopolítica do conhecimento", em que países de maior poder econômico sobressaem-se academicamente, "enunciando" e

\section{Sobre os Autores}

F.R.S.

orcid.org/0000-0002-4096-4643 Universidade de Brasília (UnB) Gama, DF

fabilation@gmail.com

\section{S.B.B.}

orcid.org/0000-0003-2829-3282

Universidade de Brasília (UnB) -

Gama, DF

barbato.silviane@gmail.com

\section{Direitos Autorais}

Este é um artigo de acesso aberto e pode ser reproduzido livremente, distribuído, transmitido ou modificado, por qualquer pessoa desde que usado sem fins comerciais. 0 trabalho é disponibilizado sob a licença Creative Commons CCBY-NC. 
tendo "enunciadas" (p. 5) suas teorias científicas. Por esta razão, pesquisadores em culturas diferenciadas tendem a adotar teorias largamente difundidas como verdades absolutas, forçando a análise dos dados a partir de teorias pré-concebidas. $O$ autor reconhece as contribuições que estas tradições trouxeram e trazem para a humanidade, mas alerta que os países que instituíram as disciplinas não detêm o "marco zero do conhecimento e que suas ideias epistêmicas não seriam o início de todas as coisas" (p. 16).

Exemplificando algumas destas generalizações, Chakkarat (2011) alega falhas de interpretação em pesquisas europeias que analisavam a produção do self, na cultura indiana. Brockmeier (2012) analisou formas diferenciadas de produção de narrativas autobiográficas em asiáticos que diferem dos cânones ocidentais na definição de self. Um exemplo de rigor necessário em análises críticas de experiências de impacto específicas é oferecido por Straub (2010), que aponta erros na interpretação e coleta de dados em estudos que equiparavam os impactos psicológicos de experiências traumáticas de descendentes de vítimas e de algozes, na Segunda Guerra.

Este discurso compartilhado entre pesquisadores culturalistas chama a atenção para a necessidade de se considerar as experiências humanas nas práticas culturais, com a utilização de metodologias que gerem inovações e ampliem as possibilidades de compreensão do humano e da produção de conhecimentos. Ressalta-se que as considerações que fazemos a estes estudos, desde a vertente Indigenous, não se relaciona com discursos acadêmicos nacionalistas, no reconhecimento de uma psicologia local e inédita, como criticado por Jahoda (2016). Como Straub (2010), reconhecemos que mesmo fenômenos inéditos são passíveis de comparação, pois a singularidade só é reconhecida graças ao pensamento comparativo, fundamental na análise científica. 0 compartilhamento de metodologias e teorias desenvolvidas entre equipes de pesquisa de diferentes culturas é compreendido como primordial para o avanço científico.

Como Straub (2010), nos interessamos por universais que nos unam enquanto humanidade. Este aprimoramento metodológico que defendemos busca evitar armadilhas evidenciadas pelo autor, com resultados que levam à hegemonizações, que podem legitimar práticas de exclusão e injustiças sociais (Straub, 2010). Este desafio pode ser mais contundente se considerarmos estudos realizados com pessoas Deficientes Intelectuais (DI) que apenas recentemente tiveram academicamente reconhecidas e difundidas suas possibilidades de desenvolvimento (American Association of Mental Retardation - AAMR, 2002). Estes indivíduos historicamente são foco de ações e discursos monológicos eugênicos, sofrendo discriminação fundada em princípios de permanência entrelaça- dos a significados de morte, de ineducabilidade, de insuperável inflexibilidade cognitiva, de um desenvolvimento sempre limitado (Davis, 2013; Goffman, 1963; Gould, 2014; Pessotti, 1984; Trent, 1994). Significados estes que se relacionam dialeticamente com outros, resultando de estudos que interpretam o humano a partir de princípios não normatizantes (Barbato-Bloch, 1997; Barríos, Barbato, \& Branco, 2012; Ferrante, 2015; Koening, 2011; Mitchell \& Snyder, 2013; Rosa, Gonzalez, \& Barbato, 2009; Vigostky, 1997). Acompanhando a historicidade do reconhecimento das possibilidades de desenvolvimento de pessoas com DI, estudos com dados qualitativos coletados com os próprios indivíduos vêm sendo desenvolvidos em várias partes do mundo (Barbato-Block, 1997; Koening, 2011; Souza \& Almeida, 2013; Stack \& MacDonald, 2014). No Brasil, estas pesquisas foram publicadas no final dos anos 1980 (Glat, 1989; Hass, 2013).

Em 2002, Nunes et al. revisaram estudos com pessoas DI e outras deficiências que coletaram dados com foco na percepção dos próprios participantes. Tendo em vista o enfoque do estudo e que fora recebido pela revista em novembro de 2001, tendo citado apenas um estudo daquele ano, decidimos enfocar a presente revisão da literatura em publicações a partir do ano 2000 a fim de disponibilizar informações sobre como estas investigações vêm sendo desenvolvidas metodologicamente.

Este trabalho contribui para o aprimoramento de desenhos metodológicos qualitativos com pessoas DI, pois, a partir das informações contidas, são possíveis inovações por meio da combinação de metodologias - levando-se em conta a discussão teórica, inclusive no diálogo de diferentes perspectivas (Lazarus, 2006). Considerando a análise dos trabalhos, ao final do texto, será proposto um desenho multimétodos.

\section{MÉTODO}

\section{FONTES DE PESQUISA E PERCURSO INICIAL}

A revisão ocorreu no período entre o segundo semestre de 2015 e o primeiro de 2016, inicialmente pelo portal da Coordenação de Aperfeiçoamento de Pessoal de Nível Superior (CAPES). A periódicos CAPES abrange a maioria das mais importantes bases de publicações nacionais e internacionais com revisão de pares e é acessível ao sistema de educação superior e órgãos governamentais brasileiros. No portal, as fontes pesquisadas foram: 1) página inicial do Portal da CAPES; 2) em separado todos os bancos de dados brasileiros; 2.a) Periódicos Eletrônicos em Psicologia (PePSIC); 2.b) IndexPsi Periódicos (INDEXPSI); 2.c) Scientific Eletronic Library on line (Scielo), 2.d) Periódico Revista Brasileira de Educação Especial (RBEE). Após a primeira fase de revisão revelar um 


\section{Hz IN PSICOLOGIA}

número reduzido de trabalhos publicados referentes ao objetivo proposto, incluímos mais duas fontes: 3) Google acadêmico; 4) Repositório Brasileiro de Teses e Dissertações RBDT, que apresenta restrição ao uso de palavras-chave, impossibilitando o refinamento da busca; e 5) contato com cinco pesquisadores brasileiros com trabalho relevante na área.

\section{CRITÉRIOS DE BUSCA, INCLUSÃO E EXCLUSÃO DE TRABA- LHOS}

Foram incluídos estudos com dados coletados diretamente com pessoas DI. As buscas foram feitas por grupos de préindexadores, palavras-chave, com menção a DI e a estudos qualitativos (ver tabela 1). Foram identificados 1149 títulos, além de 69 resumos que indicavam contemplar os objetivos da revisão. Neste processo, foram excluídos aqueles que apresentavam dados coletados apenas com pessoas que interagiam com indivíduos DI e teóricos, sobre outras deficiências ou de outros países. Por fim, foram excluídos 1129 trabalhos e selecionados 25 pesquisas empíricas. Destes trabalhos, cinco ainda foram excluídos na análise, por não deixarem claro em seus resumos se as pesquisas eram realizadas ou não com pessoas DI.

0 primeiro grupo de palavras-chave da Tabela 1 foi aplicado aos bancos de dados, no entanto, as restrições impostas quanto ao uso de caracteres resultaram na diferenciação e redução dos indexadores. Por exemplo, o banco Pepsic fazia restrição a uso de caracteres boleanos e por isto utilizamos apenas os pré-indexadores deficiência e ensino. Na Scielo, havia estudos das áreas da saúde e afins, sem menção ao discurso da pessoa DI e, por isto, restringimos a busca ao campo das ciências sociais. No Google Acadêmico, tivemos acesso também aos repositórios das universidades. Foram encontrados dois trabalhos com narrativas com pessoas DI na Universidade Federal do Rio Grande do Sul: Carneiro (2007) e Hass (2013). Dos trabalhos indicados pelos cinco pesquisadores consultados, apenas a tese de Castanheira (2014) contemplava os objetivos da revisão.

Tabela 1. Bases pesquisadas, palavras-chave tal qual utilizadas na busca, quantidade de resumos lidos, descartados e estudos selecionados

\begin{tabular}{|c|c|c|c|c|}
\hline Palavras-chave & $\begin{array}{c}\text { Estudos } \\
\text { recuperados }\end{array}$ & $\begin{array}{l}\text { Resumos } \\
\text { lidos }\end{array}$ & $\begin{array}{c}\text { Estudos } \\
\text { Descartados }\end{array}$ & Selecionados \\
\hline $\begin{array}{l}\text { CAPES: ("pessoa" OR "pessoas" OR "aluno" OR } \\
\text { "alunos" OR "estudante" OR "estudantes") AND } \\
\text { ("deficiência intelectual" OR "deficiência } \\
\text { mental") AND ("educação especial" OR "ensino } \\
\text { especial") AND ("sentido" OR "sentidos" OR } \\
\text { "percepção" OR "percepções" OR "significado" } \\
\text { OR "significados" OR "história de vida" OR } \\
\text { "estudo de caso" OR "narrativas" OR "ponto de } \\
\text { vista") }\end{array}$ & 458 & 22 & 17 & $\begin{array}{c}\text { Araújo e Aiello (2013); } \\
\text { Veltrone e Mendes (2009), } \\
\text { (2011); Souza e Almeida } \\
\text { (2013). }\end{array}$ \\
\hline Pepsic: deficiência e ensino & 62 & 4 & 4 & Ferrari e Marques (2010) \\
\hline $\begin{array}{l}\text { Index Psi: ("deficiência intelectual" OR } \\
\text { "deficiência mental") }\end{array}$ & 15 & 6 & 5 & 0 \\
\hline $\begin{array}{l}\text { Scielo: "deficiência intelectual" OR "deficiência } \\
\text { mental" }\end{array}$ & 95 & 6 & 3 & $\begin{array}{l}\text { Guhur (2007); Luiz e Kubo } \\
\text { (2007); Gomes e Rey (2008). }\end{array}$ \\
\hline $\begin{array}{l}\text { RBEE: ("deficiência intelectual" OR "deficiência } \\
\text { mental") }\end{array}$ & 195 & 5 & 2 & $\begin{array}{l}\text { Leite e Monteiro (2008); } \\
\text { Freitas e Castro (2006). }\end{array}$ \\
\hline $\begin{array}{l}\text { Google acadêmico: deficiência intelectual, } \\
\text { deficiência mental, estudo qualitativo. }\end{array}$ & 320 & 22 & 14 & $\begin{array}{l}\text { Amaral (2004); Antunes (2012); } \\
\text { Carneiro (2007); Hass (2013); } \\
\text { Oliveira (2002); Rosa (2014); } \\
\text { Santos (2006); Tomaino (2009) }\end{array}$ \\
\hline Contato com pesquisadores da área & 5 & & 4 & Castanheira (2014) \\
\hline
\end{tabular}




\section{N"INTERACÃO EM ET PSICOLOGIA}

\section{ANÁLISE}

Foi realizada a análise de conteúdo (Gomes \& Caminha, 2014; Mayring, 2000; Mozzato \& Grzybovski, 2011; Roskos, Christie, Widman, \& Holding, 2010), com reiteradas leituras, síntese e anotações sistematizadas com foco no objetivo proposto. Inicialmente, foram delimitadas as seguintes categorias temáticas para organização dos dados: área do conhecimento, perspectiva teórica, participantes, objetivos, metodologia adotada para coleta, análise dos dados e resultados. Com a leitura reflexiva de cada estudo, identificou-se informações referentes às categorias temáticas estabelecidas e foram feitas anotações descritivas. Foram excluídos trabalhos que não indicavam os sujeitos da coleta em seus resumos e que, durante a leitura reflexiva do texto completo, evidenciou-se que os participantes eram pessoas que interagiam com indivíduos DI. Após o tratamento dos dados, o conteúdo de cada estudo foi agrupado em tabela para identificação de similaridades e diferenças. Em seguida, os dados foram analisados inferencialmente e promoveu-se um diálogo dos resultados com estudos contemporâneos.

\section{RESULTADOS}

Foram selecionados vinte estudos, nove teses/dissertações e onze artigos apresentados detalhadamente na tabela 2 (em Apêndice). As categorias descrevem os estudos encontrados de acordo como formato dos trabalhos, aporte teórico, objetivos, participantes, objetivo, metodologia e resultados. Os participantes das pesquisas analisadas eram divididos entre adolescentes, jovens e adultos. Com relação ao gênero, participaram da pesquisa indivíduos de ambos os sexos. Foi percebida uma proeminência de temas de pesquisa relacionados à educação e ou escolarização da pessoa com DI. Das nove teses e dissertações, oito eram da área de educação. Dos onze artigos, divididos em áreas de psicologia e educação, quatro abordavam temáticas relacionadas à inclusão e/ou exclusão escolar da pessoa com DI. Os trabalhos restantes abordavam temáticas relacionadas a serviços sociais (Araújo \& Aiello, 2013); de fonoaudiologia (Freitas \& Castro, 2006; Leite \& Monteiro, 2008); relacionamento afetivo e sexualidade (Luiz \& Kubo, 2007; Morales \& Batista, 2010) e um estudo sobre emoções, que tinha como contexto ambiente de aprendizagem (Guhur, 2007).

Como consta na tabela 2, quinze trabalhos utilizaram entrevistas, sendo sete semiestruturadas ou estruturadas, com a introdução de objetos mediacionais de coleta. Os oito estudos restantes, metodologicamente nomeados como estudos de casos, histórias de vida, entrevistas abertas e narrativas, realizaram as entrevistas em dois ou mais encontros com os participantes, coletando os dados com a aplicação de multimétodos, introduzindo artefatos como fotografias, produção de textos, desenhos, observação etc. Os estudos de Carneiro (2007) e Santos (2006) tiveram os dados conferidos com os participantes. Por outro lado, nenhuma das pesquisas foi organizada e/ou analisada com a participação de pessoas DI. Hass (2013) organizou multimétodos individualizados para cada participante a partir de temas de interesse levantados por eles ao longo das entrevistas e desenvolveu tecnologias de comunicação para transpor as barreiras comunicativas dos participantes, ao invés de selecionar colaboradores sem comprometimentos de fala. Os trabalhos de Castanheiras (2014) e Hass (2013) evidenciaram que pessoas com DI utilizam as redes sociais para escapar à vigilância dos familiares e se comunicarem mais livremente. As pesquisas de Carneiro (2007) e Freitas e Castro (2006) trouxeram relatos de dificuldades por parte dos participantes em iniciar entrevistas de forma aberta.

Quanto à análise, os trabalhos utilizaram temas e categorias analíticas de interpretação, com caracterização e definição segundo os objetivos da pesquisa. Seis estudos definiram suas categorias analíticas antes da coleta. Os estudos de Oliveira (2002); Tomaino (2009); Veltrone e Mendes (2009); Morales e Batista (2010) utilizaram métodos quantiquali, com análises fatoriais. Todas as pesquisas tiveram seus objetivos e análises definidos e interpretados pela teoria apresentada. Todos os resultados apontam para dificuldades de inclusão da pessoa com DI na cultura brasileira, que ainda produz fortes significados sobre a capacidade limitada destes indivíduos. Quatorze estudos apontam para a superproteção familiar da pessoa com DI o que incide na manutenção da participação em espaços segregados de aprendizagem e ausência de empoderamento. Nesta revisão não havia nenhum estudo com crianças e apenas um trabalho relacionado ao estudo das emoções da pessoa com DI.

\section{DISCUSSÃO}

Pesquisas que fazem uso de multimétodos vêm sendo realizadas em várias partes do mundo, sendo reconhecidas por pesquisadores como eficazes na compreensão da complexidade dos processos humanos, difíceis de serem acessados, evitando-se reducionismos (Darbyshire, MacDougall, \& Schiller, 2005; Engel, Coll, \& Bustos, 2013; Lazarus, 2006; Levi, 1992; 1981/ 2003). A fim de se superar dificuldades que ocorrem devido às diferentes sintomatologias como, por exemplo, as decorrentes de problemas de fala, a opção tem sido o desenho de estudos de caso com desenvolvimento de tecnologias comunicativas individualizadas, buscando-se a indicação de interesses pelos próprios participantes (Hass, 2013) e instrumentos mediadores alternativos. 
Os métodos narrativos, com o uso de entrevistas semiestruturadas e multimétodos, têm buscado apoiar e incentivar os participantes com DI a narrar (Amaral, 2004; Antunes, 2012; Carneiro, 2007; Castanheira, 2014; Gomes \& Rey, 2008; Hass, 2013; Rosa, 2014; Santos, 2006). Os estudos demonstram que fazem-se necessárias a geração de técnicas que permitam temporalidades estendidas e a retomada de tópicos discutidos e replanejamento das entrevistas após estudo exploratório ou durante o processo de coleta (Carneiro, 2007; Hass, 2013; Santos 2006)

Considerando-se o histórico de estigmatização sofrido por pessoas com DI, há estudos que têm por princípio o lema "nothing about us, without us", ou seja, "nada sobre nós, sem nossa participação" (Castanheira, 2014; Stack \& MacDonald, 2014), como os que optam pela checagem de dados com os participantes (Carneiro, 2007; Santos, 2006). Embora a participação de pessoas com DI na interpretação dos resultados venha sendo desenvolvida em pesquisas em outros países (Iriarte, O'Brien, McConkey, Wolfe \& O' Doherty, 2014; Koening, 2011), no Brasil essa prática precisa ser mais difundida. 0 uso de instrumentos como este possibilita, também, a ampliação de formas de produção de significados pelos participantes.

$\mathrm{O}$ acesso mais amplo de pessoas com DI às redes sociais (Castanheira, 2014; Hass, 2013) pode gerar novos métodos que possibilitem mediar a superação de dificuldades enfrentadas em entrevistas abertas por parte dos participantes (Carneiro, 2007; Freitas \& Castro, 2006). Da mesma forma, pode contribuir para novos desenhos de entrevistas episódica (Koening, 2011) e semi estruturada.

Um dos princípios da vertente Indigenous de que as práticas culturais devem ser consideradas no desenvolvimento das pesquisas pode ser exemplificado na comparação dos estudos de Darbyshire, MacDougal e Schiller (2005) e Castanheira (2014). Em ambos os estudos, câmeras fotográficas descartáveis foram distribuídas aos participantes para que tirassem fotografias; entretanto, a anuência dos participantes foi diferente nas duas pesquisas. Enquanto essa prática foi bem sucedida no primeiro estudo mencionado, Castanheira (2014) teve pouca adesão dos participantes, uma vez que estes temiam ter as câmeras furtadas por fazerem parte de uma comunidade carente e violenta, medo este reforçado pela superproteção dos pais. Isto mostra que metodologias adotadas com sucesso em algumas culturas podem ter o caminho inverso em outras.

Estudos com objetivos pré-estabelecidas podem levar o pesquisador a desconsiderar aspectos relevantes que podem emergir durante a interação com os participantes. Esta observação foi percebida em um dos estudos (Carneiro, 2007), onde a pesquisadora relata o desejo de explorar dados que emergiram durante a investigação, mas se ateve ao foco inicial da pesquisa.

Considerando-se o objetivo da revisão, verificamos que os trabalhos revisados trazem importantes contribuições para o desenho de metodologias com narrativas de pessoas DI e informam sobre condições de socialização inclusiva, na contemporaneidade brasileira, em pesquisas que buscam compreender os processos e dinâmicas de desenvolvimento. Foram identificadas metodologias inovadoras com o uso de multimétodos qualitativos, visando o aprofundamento dos dados a fim de identificar as interpretações dos participantes sobre variados aspectos de suas vidas.

Apesar das inovações, são necessários avanços. Neste sentido, propomos incluir indivíduos DI na análise interpretativa dos dados, a exemplo dos estudos desenvolvido por Koening (2011) e Iriarte, O'Brien, McConkey, Wolfe \& O' Doherty (2014). Quanto à teorização dos processos de interpretação de si de pessoas com DI, metodologias orientadas por princípios da Teoria Fundamentada - TF (Charmaz, 2009; Glaser \& Strauss, 1967/2006) são relevantes, pois possibilitam a ampliação da perspectiva, uma vez que todos os estudos tiveram objetivos definidos e dados coletados após teorização. Os precursores desta vertente defendem que tanto objetivos quanto a revisão teórica devem ser definidos após os primeiros contatos com os participantes e entrada em campo, para que o delineamento da pesquisa seja orientado a partir do dado e para o dado, ao invés de teorias prévias (Lal, Suto \& Ungar, 2012; Ryan, 2014), promovendo a compreensão aprofundada necessária a novas teorizações. Para Glaser (2004), este procedimento impede que a pesquisa parta de problemas preconcebidos, de modo que o pesquisador deve ouvir o participante ao invés de induzi-lo a falar sobre assuntos que podem lhe ser de pouco interesse.

Estudos em TF defendem que a coleta deve começar por meio de entrevistas abertas, através das quais a pessoa elege o que é importante ao invés de ser dirigida pelo pesquisador. Apesar de este trabalho trazer dados que sugerem que pessoas com DI sentem dificuldade em realizar entrevistas abertas, a tentativa é válida, uma vez que o problema pode não ser generalizado a todos os indivíduos com DI, sobretudo àqueles que se desenvolvem em condições de socialização diferenciadas daquelas apresentadas nos estudos desta revisão.

Como alternativa para esta possível dificuldade, sugerimos um roteiro de entrevista inicial aberto, com mais de um encontro com os participantes em seus locais de preferência e o uso de outras ferramentas de comunicação, como a internet e redes sociais. Os primeiros encontros com os participantes seriam norteadores das coletas posteriores, a partir dos temas emergentes, que seriam aprofundados. Neste pro- 


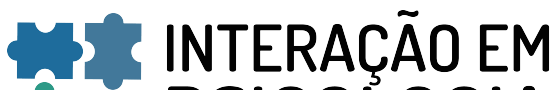 ET PSICOLOGIA}

cesso, realizar coleta multimétodos que podem ser refinadas, considerando-se a peculiaridade de cada participante.

Levando-se em conta que a maioria dos estudos traziam teorias, dados e resultados que apontam para a dificuldade de inclusão de pessoas com $\mathrm{DI}$, devido à reprodução social de significados relacionada à capacidade limitada de desenvolvimento destes indivíduos, sugerimos trabalhos sobre emoções da pessoa com DI. Neste sentido, esperamos desenvolver pesquisa empírica sobre processos de emoções da pessoa com DI em ambientes inclusivos em relação simultânea com Coping e/ou auto regulação, enfocando a forma com que estes indivíduos lidam com suas demandas adaptativas, appraisal e/ou ressignificação das emoções, entendidas como significados atribuídos a emoções decorrentes de relações/experiências interpessoais e ou ambientais (Lazarus, 2006; Valsiner, 2005). O trabalho será orientado por alguns pressupostos da TF a fim de desenvolver entrevistas abertas e aprofundar a fundamentação teórica após os primeiros contatos com os participantes.

Pesquisas em emoções realizadas por meio de narrativas têm se mostrado desafiadoras em todas as etapas do estudo, pois devem trazer à tona os componentes subjetivos (Lazarus, 2006; Scherer, 2005). Neste sentido, compreende-se como uma limitação deste trabalho a incidência de poucos estudos voltados para as emoções da pessoa com DI. Considerando-se a amplitude desta revisão, que abarcou os últimos 16 anos da produção brasileira, em seguida ela pode ser ampliada para estudos internacionais, em diferentes culturas e restrita aos 5 anos anteriores ao início da revisão.

Desta forma, práticas metodológicas podem continuar a ser aprimoradas. No entanto, elas devem ser aperfeiçoadas com o olhar crítico proposto neste trabalho, tendo em vista a defesa mais relevante da vertente cultural Indigenous, que é reconhecer a influência dos contextos culturais na aplicação de metodologias e interpretações na busca de avanços no conhecimento científico.

\section{CONTRIBUIÇÃO DE CADA AUTOR}

F.R.S. e S.B.B. desenvolveram a conceitualização, a metodologia e revisaram e editaram a redação; F.R.S. preparou o rascunho original, realizou a tabulação dos dados e a visualização; e S.B.B. supervisionou.

\section{DECLARAÇÃO DE CONFLITOS DE INTERESSES}

Os autores declaram que não há conflitos de interesses no presente artigo.

\section{DECLARAÇÃO DE FINANCIAMENTO}

Fabíola Ribeiro de Souza é pesquisadora financiada pela Secretaria de Estado de Educação do Distrito Federal SEEDF, recebeu recursos do Fundo de Apoio a Pesquisa do Distrito Federal - FAP DF e do Decanato de Apoio a Pesquisa e Pós-Graduação da Universidade de Brasília - DPP/UNB.

Silviane Bonaccorsi Barbato é bolsista CNPq.

\section{REFERÊNCIAS}

Amaral, T. P. D. (2004). Deficiência mental leve: Processos de escolarização e de subjetivação (Tese de doutorado). Universidade de São Paulo, São Paulo.

Associação Americana de Retardo Mental (2002). Retardo mental: Definição, classificação e sistemas de apoio (A. Cheuiche, Trad.). Porto Alegre: ArtMed.

Antunes, K. C. V. (2012). História de vida de alunos com deficiência intelectual: Percurso escolar e a constituição do sujeito (Tese de doutorado). Universidade do Estado do Rio de Janeiro, Rio de Janeiro.

Araújo, G. M. S., \& Aiello, A. L. (2013). Rede social de apoio de mães com deficiência intelectual. Psicologia: Reflexão e Crítica, 26 (4),752-761. http://dx.doi.org/10.1590/S010279722013000400016

Atkinson, R. (1988). The life story interview. Thousand Oaks: Sage.

Barbato-Bloch, S. (1997). Produção textual de uma jovem com Síndrome de Down: Explorando novos espaços discursivos (Tese de doutorado). Universidade de Brasília, Brasília-DF

Barríos, A., Barbato, S. B., \& Branco, A. M. C. U. de A. (2012) El analisis microgenético en el estudio del desarrollo moral. Revista de Psicología, 30, 249-279.

Brockmeier, J. (2012). Localizing oneself: Autobiographical remembering, cultural memory and the Asian American experience. Em J. Brockmeier (Ed.) Beyond the archive: memory, narrative and the autobiographical process (pp. 121-133). Oxford: Blackwell.

Bruner, J. (1997). Atos de Significação (S. Costa, Trad.). Porto Alegre: ArtMed.

Carneiro, M. S. C. (2007). Deficiência mental como produção social: Uma discussão a partir da história de vida de adultos com Síndrome de Down (Dissertação de mestrado). Universidade Federal do Rio Grande do Sul, Porto Alegre.

Castanheira, A. A. C. (2014). "Deixa que eu falo": A inclusão sob a ótica do estudante com deficiência intelectual (Dissertação de mestrado). Universidade Federal do Rio de Janeiro, Rio de Janeiro. 


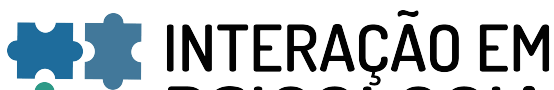 ET PSICOLOGIA}

Chakkarath, P. (2011). The role of indigenous psychologies in the building of basic cultural psychology. Em J. Valsiner (Ed.). The oxford handbook of cultural psychology. (pp.7195). Oxford: OUP.

Charmaz, K. (2009). A construção da teoria fundamentada (J. E. Costa, Trad.). Porto Alegre: ArtMed.

Darbyshire, P., MacDougall. C., \& Schiller, W. (2005). Multiple methods in qualitative research with children: More insight or just more? Qualitative research, 5(4), 417-436. http://dx.doi.org/10.1177/14687941050569

Davis, L. J. (2013). Introduction: Disability, normality and power. Em L. J. Davis (Ed.), The disability studies reader. (pp. 01-16). London: Routledge.

Engel, A., Coll, C., \& Bustos, A. (2013). Distributed teaching presence and communicative patterns in asynchronous learning: Name versus reply networks. Computer and Education, 60, 189-196. http://dx.doi.org/10.1016/j.compedu.2012.06.011

Ferrante, C. (2015). Discapacidad y mendicidad en la era de la Convención: ¿Postal del pasado? Convergencia Revista de Ciencias Sociales, 68, 151-176.

Ferrari, A., \& Marques, L. P. (2010). Homossexualidade e deficiência mental: Jogos discursivos e de poder na construção dessas identidades no contexto escolar. Revista Mal Estar e Subjetividade, 10(2), 653-672.

Freitas, A. P. de, \& Castro, G. (2006). A constituição de processos dialógicos em um grupo de jovens com deficiência mental. Revista Brasileira de Educação Especial, 12(1), 4964.

Glat, R. (1989). Somos iguais a vocês: Depoimentos de mulheres com deficiência mental. Rio de Janeiro: Agir.

Glaser, B. G., \& Strauss, A. L. (1967/2006). The discovery of Grounded Theory. New York: Aldine de Gruyter.

Glaser, B. G. (2004). Remodeling Grounded Theory. Forum Qualitative Sozialforschung/Forum: Qualitative Social Research, 5(2), 1-15.

Goffman, E. (1963). Stigma. London: Penguin.

Gomes, I. S., \& Caminha, I. de O. (2014). Guia para estudos de revisão sistemática: Uma opção metodológica para as ciências do movimento humano. Movimento, 20(1), 395411.

Gomes, C., \& Rey, L. F. G. (2008). Psicologia e inclusão: aspectos subjetivos de um aluno portador de deficiência mental. Revista Brasileira de Educação especial, 14(1), 52-62.

Gould, S. J. (2014). A falsa Medida do Homem (V. L. Siqueira, Trad.). São Paulo: Martins Fontes.

Guhur, M. de L. P. (2007). A manifestação da afetividade em sujeitos jovens e adultos com deficiência mental: Perspectivas de Wallon e Bakhtin. Revista Brasileira de Educação Especial, 13(3), 381-398.
Hass, C. B. (2013). Narrativas e percursos escolares de jovens e adultos com deficiência: "Isso me lembra uma história!" (Dissertação de mestrado). Universidade Federal do Rio Grande do Sul, Porto Alegre.

Iriarte, G.E., O'Brien, P., McConkey, R., Wolfe M., \& O'Doherty, S. (2014). Identifying the key concerns of Irish persons with intellectual disability. Journal of Applied Research in Intellectual Disabilities, 27, 564-575. http://dx.doi.org/10.1111/jar.12099

Jahoda G. (2016). On the rise and the decline of "Indigenous Psychology". Culture \& Psychology, 22(2) 169-181. http://dx.doi.org/10.1177/1354067X16634052

Koening, O. (2011). Any added value? Co-constructing life stories of and with people with intellectual disabilities. British Journal of Learning Disabilities, 40(3), 213-221. http://dx.doi.org/10.1111/j.1468-3156.2011.00695.x

Lal, S., Suto, M., \& Ungar, M. (2012). Examining the potential of combining methods of Grounded Theory and narrative inquiry: A comparative analysis. The Qualitative Report, 17(41), 1-22.

Lazarus, R. S. (2006), Emotions and interpersonal relationships: Toward a person-centered conceptualization of emotions and coping. Journal of Personality, 74, 9-46. http://dx.doi.org/10.1111/j.1467-6494.2005.00368.x

Leite, G. A., \& Monteiro, M. I. B. (2008). A construção da identidade de sujeitos deficientes no grupo terapêutico-fonoaudiológico. Revista Brasileira de Educação Especial, 14(2), 189-200.

Levi, G. (1992). II piccolo, il grande, il piccolo: Intervista a Giovanni Levi. Percorsi di Ricerca. Meridiana, 10, 2011-234.

Levi, G. (2003). Un problema de escala. Relaciones. Estudíos de História y Sociedad, 24(95), 279-288. (Original publicado em 1981)

Luiz, E. C., \& Kubo, O. M. (2007). Percepções de jovens com Síndrome de Down sobre relacionar-se amorosamente. Revista Brasileira de Educação Especial, 13(2), 219-238.

Mayring, Philipp (2000). Qualitative Content Analysis. Forum Qualitative Sozialforschung / Forum: Qualitative Social Research, 1(2): 1-10, jun.

McAdams, D. P., \& Guo, J. (2015). Narrating the generative life. Psychological Science, 26, 475-483. http://dx.doi.org/ $10.1177 / 0956797614568318$

Mignolo, W. D. (2009). Epistemic disobedience, independent though and de-colonial freedom. Theory, Culture \& Society, 26(7-8), 1-23.

Mitchell, D., \& Snyder, S. (2013). Narrative prosthesis. Em L. J. Davis (Ed.), The disability studies reader. (pp. 222-235). London: Routledge.

Morales, A. S., \& Batista, C. G. (2010). Compreensão da sexualidade por jovens com deficiência intelectual. Psicologia Teoria e Pesquisa, 26(2), 234-244. 


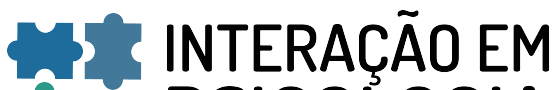 ET PSICOLOGIA}

Mozzato, A. R., \& Grbovisky, D. (2011). Análise de conteúdo como técnica de análise de dados qualitativos no campo da administração: potencial e desafios. Revista de Administração Contemporânea - RAC, 15(4), 731-747.

Nunes, L. R. O. de P., Glat, R., Ferreira, J. R., Mendes, E. G., de Paula, K. P., \& Nogueira, M. L. (2002). O que revelam as teses e dissertações sobre a autopercepção do portador de necessidades especiais? Temas em Psicologia, 10(2), 136153.

Oliveira, A. A. S. (2002). Representações sociais sobre educação especial sobre educação especial e deficiência: O ponto de vista de alunos deficientes e professores Eepecializados (Tese de doutorado). Universidade Estadual Paulista, Marília.

Pessoti, I. (1984). Da superstição à ciência. São Paulo: Tao Edusp.

Rosa, A., Gonzalez, M. F., \& Barbato, S. (2009). Constuyendo narraciones para dar sentido a experiencias vididas: Um estúdio sobre las relaciones entre la forma de las narraciones y el posicionamiento personal. Estudios de Psicologia, $30(2)$, 231-259. http://dx.doi.org/10.1174/021093909788347118

Rosa, F. G. S. (2014). Educação e inclusão da pessoa com deficiência intelectual no mundo do trabalho: 0 caso dos egressos do centro de ensino especial Helena Antipoff (Dissertação de mestrado). Universidade Federal do Maranhão, São Luis.

Roskos, K.A.; Christie, J.F.; Widman, S., \& Holding, A. (2010). Three decades in: primimg for meta-analysis in play -literacy research. Journal of Early Childhood Literacy, 10(1), 55-96. http://dx.doi.org/10.1177/1468798409357580

Ryan, J. (2014). Uncovering the hidden voice: Can Grounded Theory capture the views of a minority group? Qualitative Research, 14, 549-566. http://dx.doi.org/10.1177/1468794112473494

Santos, R. A. (2006). Processos de escolarização e deficiência: Trajetórias singulares de ex-alunos de classe especial para deficiência mental (Tese de doutorado). Pontifícia Universidade Católica de São Paulo, São Paulo.

Scherer, K. R. (2005). What are emotions and how can they be measured? Social Science information, 44(4), 695-729. http://dx.doi.org/10.1177/0539018405058216

Souza, F. R., \& Almeida, S. F. C. (2013). A inclusão/exclusão escolar: Concepções de pais e de jovens alunos com deficiência intelectual. Interacções, 26(9), 289-310.
Stack, E., \& MacDonald, K. E. (2014). Nothing about us without us: Does action research in developmental disabilities research measure up? Journal o Policy and Practice in Intellectual Disability, 11(2), 83-91.

Straub, J. (2010). Understanding transgenerational transmission: The burden of history in families of Jewish victims and their National socialist victimizers. Em J. Straub \& J. Rüsen (Eds.), Dark traces of the past: Psychoanalysis and historical thinking. New York: Berghahn Books.

Straub, J. (2013). Personal identity: A concept in humanist tradition. Em J. Rüsen (Ed.), Approaching humankind: Towards an intercultural humanism. VeR Unipress: Taywan.

Tomaino, G. C. (2009). Do ensino especializado a educação de jovens e adultos: Análise das trajetórias escolares nas perspectivas dos alunos, familiares e professores (Dissertação de mestrado). Universidade Estadual Paulista, Araraquara.

Trent, J. W. (1994). Inventing feeble mind: A history of mental retardation in the United States. Berkeley: University of California Press.

Valsiner, J. (2005). Soziale und emotionale Entwicklungsaufgaben im Kulturellen Kontext. Em J. Asendorpf \& H. Rauh (Eds), Enziklpadie der Psychologie. V.3. Gottingen: Hogrefe.

Veltrone, A. A., \& Mendes, E. G. (2009). Inclusão e fracasso escolar: $O$ que pensam os alunos com deficiência mental?Revista Educação Especial, 22(33), 59-71.

Veltrone, A. A., \& Mendes, E. G. (2011). Percepções dos alunos com deficiência intelectual sobre sua matrícula na classe especial e comum. Revista Eletrônica de Educação, 5(2), 59-81.

Vigotsky, L. S. (1997). Los problemas fundamentales de la Defectología contemporánea. (Vol. V). Madrid: Visor.

Recebido: em 29/08/2016

Primeira decisão editorial em: 11/02/2017

Aceito em: 07/03/2017

Nota. As autoras agradecem a Prof. ${ }^{a}$ Cátia Candido da Silva por suas contribuições de revisão textual e das normas da APA. 
Tabela 2. Resultados descritivos dos estudos encontrados

\begin{tabular}{|c|c|c|c|c|}
\hline $\mathbf{N}$ & $\begin{array}{c}\text { Autor } \\
\text { Formato } \\
\text { Aporte Teórico }\end{array}$ & $\begin{array}{l}\text { Participante } \\
\text { Sexo } \\
\text { Idade }\end{array}$ & Método & $\begin{array}{l}\text { Procedimento de coleta } \\
\text { Instrumentos }\end{array}$ \\
\hline 7 & $\begin{array}{l}\text { Amaral } \\
\text { (2004) } \\
\text { Tese } \\
\text { Histórico cultural/ } \\
\text { histórico crítica no } \\
\text { campo da DI }\end{array}$ & $\begin{array}{c}2 \mathrm{~F} \\
31 \text { a } 34\end{array}$ & $\begin{array}{l}\text { Estudo de } \\
\text { caso }\end{array}$ & $\begin{array}{l}\text { Dados coletados por registros médicos e escolares dos participantes, de } 2 \text { a } 3 \\
\text { entrevistas semiestruturadas com produção de textos pelos participantes e } \\
\text { observação direta, a fim de se averiguar processos de subjetivação da } \\
\text { Deficiência Mental de alunas egressas de classes especiais. Roteiro de } \\
\text { entrevista organizado a partir das informações dos documentos, a fim de } \\
\text { auxiliar o resgate da memória dos participantes, com foco no período de } \\
\text { escolarização de alunas egressas da educação exclusivamente especial. } \\
\text { Registro de novos arquivos ou objetos entregues ao pesquisador pelos } \\
\text { participantes. Entrevistas posteriores com roteiros flexíveis, orientadas pelos } \\
\text { temas emergentes dos encontros. }\end{array}$ \\
\hline 2 & $\begin{array}{l}\text { Antunes } \\
(2012) \\
\text { Tese } \\
\text { Abordagem } \\
\text { psicossocial da } \\
\text { deficiência }\end{array}$ & $\begin{array}{c}5 \\
3 F 2 M \\
13 \text { a } 23\end{array}$ & $\begin{array}{l}\text { História de } \\
\quad \text { vida }\end{array}$ & $\begin{array}{l}\text { História de vida, organizada por meio de entrevistas abertas com foco em } \\
\text { experiências de inclusão escolar. }\end{array}$ \\
\hline 3 & $\begin{array}{l}\text { Araújo e Aiello } \\
\qquad(2013) \\
\text { Artigo } \\
\text { Rede de apoio a } \\
\text { mães com DI }\end{array}$ & $\begin{array}{c}5 \\
F \\
28 \text { a } 42\end{array}$ & Entrevista & $\begin{array}{l}\text { Entrevistas estruturadas a fim de caracterizar a rede pessoal de apoio a mães } \\
\text { com DI e analisar opinião dos fornecedores pelo apoio. Foram feitas } \\
\text { entrevistas com as mães de caracterização e aspectos sócio demográficas. } \\
\text { Entrevistas sobre o apoio recebido por meio de cartões de resposta e } \\
\text { representações gráficas que ilustravam as dimensões da pesquisa }\end{array}$ \\
\hline 4 & $\begin{array}{l}\text { Carneiro } \\
\text { (2007) } \\
\text { Tese } \\
\text { Histórico cultural/ } \\
\text { Vigotsky }\end{array}$ & $\begin{array}{c}3 \\
2 \mathrm{M} 1 \mathrm{~F} \\
31 \text { a } 43\end{array}$ & $\begin{array}{l}\text { Entrevistas } \\
\text { abertas } \\
\text { com roteiro } \\
\text { prévio }\end{array}$ & $\begin{array}{l}\text { Coletas realizadas por meio de entrevistas abertas, em mais de um encontro } \\
\text { entre pesquisadora e pesquisado, com uso de documentos e informações } \\
\text { dadas por amigos e familiares. Roteiro de entrevista e coleta com foco no } \\
\text { período de escolarização, eventos marcantes e dificuldades. Dados gravados, } \\
\text { transcritos e transformados em narrativas, suprimindo repetições e digressões } \\
\text { vistas como impertinentes e mantendo marcas de expressão oral e trechos } \\
\text { dos diálogos. Dados conferidos com um dos participantes. }\end{array}$ \\
\hline 5 & $\begin{array}{l}\text { Castanheiras } \\
(2014) \\
\text { Dissertação } \\
\text { Bakhtin, Vigotsky e } \\
\text { Focault }\end{array}$ & $\begin{array}{c}5 \\
3 F 2 M \\
14 \text { a } 27\end{array}$ & $\begin{array}{l}\text { História } \\
\text { oral/ } \\
\text { narrativa } \\
\text { sobre } \\
\text { situação } \\
\text { presente }\end{array}$ & $\begin{array}{l}\text { Entrevistas abertas, com sessões semiestruturadas sobre processo de } \\
\text { inclusão escolar e social de alunos com DI matriculados em escola regular. } \\
\text { Entrega de câmeras descartáveis para que os alunos tirassem fotos dos } \\
\text { ambientes que se sentiam incluídos ou não. Pesquisa documental sobre } \\
\text { movimentos de vida independente de pessoas com DI no Brasil e no mundo }\end{array}$ \\
\hline
\end{tabular}

\section{Interpretação/ análise dos dados}

Eixos temáticos organizados a partir dos relatos, com recorte final baseado nas perguntas de pesquisa. Formação de quadro analítico comparativo das histórias dos participantes. Dados interpretados a partir do referencial teórico e de pesquisas na área. Resultados apontam para processos de incorporação de crenças sobre incapacidade e culpabilidade do fracasso escolar produzidos na relação com educadores e profissionais de saúde.

Formação de categorias de análise temática que emergiram durante e após a coleta. Análise interpretativa dos dados, incluindo contexto histórico e a teoria referendada. Os resultados demonstraram que a trajetória dos alunos na inclusão escolar foi marcada pelo descrédito dos que os assistem, sendo que na prática este sistema prima apenas por uma reorganização escolar.

Análise fatorial a partir dos dados coletados nas entrevistas e comentários feitos pelas participantes trazendo indícios de necessidades de melhor apoio para pais e mães com DI

Análise interpretativa centrada nos objetivos e teoria defendida, apontando que a constituição de pessoas com $\mathrm{DI}$, apesar das adversidades, se dá na relação com as demais pessoas.

Dados transcritos e lidos permitindo a formação de categorias analíticas dos temas emergentes no discurso dos entrevistados. As fotos também formaram outras categorias. Os dados foram interpretados pela teoria referendada, atestando que os participantes não participam de movimentos de inclusão, sugerindo temas para novas investigações e políticas públicas com pessoas DI. 
Tabela 2. Resultados descritivos dos estudos encontrados (continuação)

\begin{tabular}{|c|c|c|c|c|c|}
\hline $\mathbf{N}$ & $\begin{array}{c}\text { Autor } \\
\text { Formato } \\
\text { Aporte Teórico }\end{array}$ & $\begin{array}{l}\text { Participante } \\
\text { Sexo } \\
\text { Idade }\end{array}$ & Método & $\begin{array}{l}\text { Procedimento de coleta } \\
\text { Instrumentos }\end{array}$ & Interpretação/ análise dos dados \\
\hline 6 & $\begin{array}{l}\text { Freitas e Castro } \\
\qquad(2006) \\
\text { Artigo } \\
\text { Sócio-histórica Bakhtin }\end{array}$ & $\begin{array}{c}4 \\
2 M 2 F \\
15 \text { a } 24\end{array}$ & Observação & $\begin{array}{l}\text { Vídeo gravação de seções semanais de fonoaudiologia, por um período } \\
\text { de } 4 \text { meses, com foco nas interações dialógicas entre } 4 \text { pessoas com } \\
\text { DI e o fonoaudiólogo, a fim de caracterizar os diferentes modos de } \\
\text { atuação do terapeuta/interlocutor e de compreender como as } \\
\text { intervenções terapêuticas contribuem para o desenvolvimento da } \\
\text { linguagem dos sujeitos. }\end{array}$ & $\begin{array}{l}\text { Dados transcritos, sendo elevados a categorias aqueles que se relacionavam } \\
\text { com a dinâmica dialógica estabelecida pelo grupo. Os dados foram } \\
\text { interpretados pela teoria referendada e apontam para a atuação do terapeuta } \\
\text { no sentido de disponibilizar para o grupo atividades significativas e variadas } \\
\text { para o acontecimento da linguagem. }\end{array}$ \\
\hline 7 & $\begin{array}{l}\text { Ferrari e Marques } \\
\qquad(2010) \\
\text { Artigo } \\
\text { Lógica Foucaltiana de } \\
\text { jogos discursivos de } \\
\text { poder e da identidade }\end{array}$ & $\begin{array}{l}1 \\
M\end{array}$ & $\begin{array}{l}\text { Conversa } \\
\text { informal }\end{array}$ & Observação e conversa informal com uma criança DI & $\begin{array}{l}\text { Análise centrada na teoria referendada, tendo o discurso do participante } \\
\text { apenas como contextualização da discussão, apontando injustiças e } \\
\text { propondo uma educação que considere as diferenças. }\end{array}$ \\
\hline 8 & $\begin{array}{l}\text { Gomes e Rey } \\
\qquad(2008) \\
\text { Artigo } \\
\text { Teoria da Subjetividade } \\
\text { de González Rey }\end{array}$ & $\begin{array}{c}1 \\
M \\
16\end{array}$ & $\begin{array}{l}\text { Estudo de } \\
\text { caso }\end{array}$ & $\begin{array}{l}\text { Observação do ambiente escolar durante sete meses, entrevista } \\
\text { semiestruturada, diários de pesquisa a fim de explorar as } \\
\text { configurações de sentido de um aluno com DI acerca do processo de } \\
\text { inclusão escolar. }\end{array}$ & $\begin{array}{l}\text { As informações obtidas foram organizadas em indicadores significativos, } \\
\text { que buscaram avançar na compreensão dos sentidos subjetivos. Os dados } \\
\text { apontaram para barreiras enfrentadas pelo aluno no que diz respeito à } \\
\text { organização simbólica da instituição escolar que, atrelada aos padrões } \\
\text { massificadores do desenvolvimento humano, se estruturava como uma } \\
\text { prática social e compensatória ao invés de formadora, desconsiderando } \\
\text { adequadamente a singularidade do aluno. }\end{array}$ \\
\hline 9 & $\begin{array}{l}\text { Guhur } \\
\text { (2007) } \\
\text { Artigo }\end{array}$ & $\begin{array}{c}16 \\
9 \mathrm{M} 7 \mathrm{~F} \\
13 \text { a } 32\end{array}$ & $\begin{array}{l}\text { Observação } \\
\text { microgené- } \\
\text { tica }\end{array}$ & $\begin{array}{l}\text { Vídeo gravação e diário de campo para registrar episódios interativos } \\
\text { de pessoas com DI durante } 22 \text { encontros de participação em um } \\
\text { programa de atividades pedagógicas alternativo ao escolar. } 0 \text { intuito } \\
\text { era caracterizar a forma como os participantes exteriorizavam suas } \\
\text { emoções nos relacionamentos e como utilizavam recursos e/ou } \\
\text { instrumentos simbólicos no processo. }\end{array}$ & $\begin{array}{l}\text { Recorte das cenas que apresentavam condutas, comportamentos e } \\
\text { verbalizações dos sujeitos participantes. Transcrição e leitura destes dados, } \\
\text { com foco nos episódios que pudessem servir como indicadores da } \\
\text { exteriorização das emoções. Dados agrupados em três conjuntos temáticos } \\
\text { e interpretados a partir da teoria referendada, apontando que os participantes } \\
\text { se constituem como interlocutores atentos, ativos e expressivos, capazes de } \\
\text { compartilhar os sentidos pretendidos nas interações com seus pares, com } \\
\text { exceção da dificuldade expressa pelos que apresentam pouco domínio } \\
\text { linguístico e, portanto, limitados recursos simbólicos. }\end{array}$ \\
\hline 10 & $\begin{array}{l}\text { Hass } \\
(2013) \\
\text { Dissertação } \\
\text { Abordagem científica } \\
\text { do pensamento } \\
\text { sistêmico de Gregory } \\
\text { Bateson e Humberto } \\
\text { Maturana }\end{array}$ & $\begin{array}{c}3 \\
2 \mathrm{M} 1 \mathrm{~F} \\
20 \text { a } 22\end{array}$ & $\begin{array}{l}\text { História } \\
\text { oral na } \\
\text { construção } \\
\quad \text { de } \\
\text { narrativas }\end{array}$ & $\begin{array}{l}\text { Foram realizados de quatro a cinco encontros com os participantes, } \\
\text { sendo dois informais, com foco na trajetória escolar de pessoas com DI } \\
\text { matriculados na Educação de Jovens e Adultos - EJA. A partir dos } \\
\text { primeiros encontros, que foram feitos por meio de entrevistas abertas, } \\
\text { a pesquisadora organizou as coletas posteriores. Foram introduzidos } \\
\text { jogos simbólicos, ou seja, métodos diversificados e individualizados a } \\
\text { partir dos temas levantados pelos participantes e as dificuldades de } \\
\text { comunicação. Foram disponibilizados aos participantes figuras, textos, }\end{array}$ & $\begin{array}{l}\text { Leituras dos dados. Categorias temáticas organizadas após as entrevistas, } \\
\text { sem hipóteses prévias, a partir do que os entrevistados elegeram como } \\
\text { importante. Análises dos sentidos compartilhados entre os participantes e } \\
\text { marcas individuais. Os dados foram analisados à luz da teoria referendada } \\
\text { trazendo diversos temas de investigação, como por exemplo, a importância } \\
\text { de processos de comunicação que garantam a estas pessoas a participação } \\
\text { social; o fracasso escolar como marco no processo de estigmatização do eu; } \\
\text { políticas públicas educacionais deficitárias; entre outras questões. }\end{array}$ \\
\hline
\end{tabular}


Tabela 2. Resultados descritivos dos estudos encontrados (continuação)

\begin{tabular}{ccccc}
\hline $\mathbf{N}$ & $\begin{array}{c}\text { Autor } \\
\text { Formato } \\
\text { Aporte Teórico }\end{array}$ & $\begin{array}{c}\text { Participante } \\
\text { Sexo } \\
\text { Idade }\end{array}$ & Método & $\begin{array}{c}\text { Procedimento de coleta } \\
\text { Instrumentos }\end{array}$ \\
\hline 11 & Leite e Monteiro(2008) & 7 & Observação & Observação de filmagens de reuniões de interação entre pessoas com \\
& Artigo & $5 \mathrm{M} 2 \mathrm{~F}$ & de banco & Dl e fonoaudiólogos. \\
& Histórico-cultural: & 16 a 27 & de dados & \\
Vigotsky e Bakhtin & & & &
\end{tabular}

Vigotsky e Bakhtin

$$
\text { de banco }
$$

12

\section{Rosa}

(2014)

Dissertação

Materialismo histórico

dialético

13 Luiz e Kubo (2007)

Artigo

Bakhtin e Wallon

Morales \& Batista

(2010)

Artigo

Histórico - cultural/

sociocultural

$\begin{array}{cr}\text { Oliveira } & 12 / \mathrm{x} \\ (2002) & 30 \text { a } 35\end{array}$

Tese

Representações

sociais e

Sócio histórico 18 a 28

sobre
Estudo de Observação não participante e entrevista semi estrutura a fim de

analisar fatores que interagem para inclusão e exclusão de pessoa com DI no mundo do trabalho.

Entrevista Coleta por meio de entrevista estruturada com roteiro organizado a $2 \mathrm{~F} 3 \mathrm{M}$ estruturada partir de levantamento de variáveis constituintes do comportamento "relacionar-se amorosamente". Entrevista individual com diferentes

percepções temáticas a fim de identificar percepções de pessoas com Síndrome de Down sobre relacionar-se amorosamente. Dados gravados e transcritos.

Observação Vídeo gravação de aulas sobre orientação sexual a fim de apontar

Inter- indícios sobre o processo de apropriação de conhecimentos e valores

relações sobre sexualidade de adolescentes e jovens, indicando aspectos do

complexas contexto que favorecem ou dificultam essas apropriações. Diário de

e campo com resumo das dinâmicas das atividades, participação dos

dinâmicas alunos e acontecimentos relevantes.

Entrevista Elaboração de categorias analíticas anteriores à coleta. Roteiro de entrevista norteado pelos objetivos da pesquisa.

\section{Interpretação/ análise dos dados}

Análise microgenética de perspectiva histórico-cultural com transcrição e

exame pormenorizado da fala dos participantes, turno a turno, com o intuito de identificar os episódios que manifestassem histórias e sentimentos a respeito da autoimagem dos participantes. Análise posterior dos dados para se identificar aspectos relevantes para o atendimento fonoaudiológico do deficiente, buscando transformações na autoimagem, decorrentes da construção da linguagem no grupo. 0 estudo demonstrou que para se transformar a imagem que os sujeitos deficientes mentais têm de si mesmos, são necessárias ações que incidam sobre o grupo social de inserção da pessoa com DI.

Transcrição dos dados e análise de conteúdo para buscar núcleos de sentidos nas concepções sobre deficiência, inclusão e exclusão. Os resultados apontam para uma reprodução educacional das demandas de mercado, influência materna nas relações de trabalho e das concepções sobre deficiência nas ações de exclusão e inclusão no emprego.

Dados organizados de acordo com os conjuntos de variáveis propostos na pesquisa. Agrupamento das verbalizações em 6 categorias temáticas por similaridades dos significados. Dados representados em quadros temáticos. Os resultados demonstram que pessoas com DI apresentam percepções similares a outros jovens e que relacionamentos amorosos se desenvolvem pelas oportunidades de se comportarem sob contingências que favoreçam estas relações.

Seleção de cinco aulas para análise. Leitura dos dados transcritos; definição de categorias temáticas norteadas pelas perguntas de pesquisa; organização de episódios definidos por sequencia de diálogos e atividades com unidades temáticas. Os resultados apontam para diferentes formas de apropriação de conceitos sobre sexualidade e para capacidade de assimilação de valores e regras culturalmente estabelecidas.

Análise analítica por meio de sistemas de categorias. Dados interpretados à luz da teoria apontando para a dificuldade de alunos e professores de transformarem suas realidades objetivas devido à ausência de aprendizagem e reflexão histórica. 
Tabela 2. Resultados descritivos dos estudos encontrados (continuação)

\begin{tabular}{|c|c|c|c|c|c|}
\hline $\mathbf{N}$ & $\begin{array}{c}\text { Autor } \\
\text { Formato } \\
\text { Aporte Teórico }\end{array}$ & $\begin{array}{l}\text { Participante } \\
\text { Sexo } \\
\text { Idade }\end{array}$ & Método & $\begin{array}{l}\text { Procedimento de coleta } \\
\text { Instrumentos }\end{array}$ & Interpretação/ análise dos dados \\
\hline 16 & $\begin{array}{l}\text { Santos } \\
(2006) \\
\text { Tese } \\
\text { Sociologia da } \\
\text { educação Pierre } \\
\text { Bordieu e Bernard } \\
\text { Lahine }\end{array}$ & $\begin{array}{c}5 \\
3 F 2 M \\
22 \text { a } 29\end{array}$ & $\begin{array}{l}\text { Entrevista } \\
\text { semiestru- } \\
\text { turada }\end{array}$ & $\begin{array}{l}\text { A fim de conhecer o percurso biográfico de pessoas com DI e } \\
\text { experiências socializadas, em especial da escola, foram realizadas, } \\
\text { pelo menos, três entrevistas com participantes que não apresentassem } \\
\text { problemas de comunicação. Roteiro de entrevista semiestruturada com } \\
\text { coleta adaptada às dificuldades de memória dos participantes que } \\
\text { tiveram liberdade para recorrer a fotos, fazer perguntas a outras } \\
\text { pessoas e consultar documentos pessoais. Perguntas organizadas a } \\
\text { partir do referencial teórico. Foram pedidas fotos aos participantes e foi } \\
\text { feita ficha de identificação para coleta com a família e nas escolas que } \\
\text { eles frequentavam. Dados transcritos e organizados biograficamente. } \\
\text { As transcrições foram feitas e lidas para os participantes, logo após os } \\
\text { encontros, a fim de aprofundar dados e obter sua aprovação. }\end{array}$ & $\begin{array}{l}\text { Dados organizados em três grandes eixos, norteados pela teoria referendada } \\
\text { e organizados antes da coleta. Tópicos emergentes dos eixos, a partir dos } \\
\text { dados coletados. Os resultados apontam que mesmo frequentando classes } \\
\text { especiais os sujeitos da pesquisa apresentam diferentes processos de } \\
\text { escolarização. }\end{array}$ \\
\hline 17 & $\begin{array}{l}\text { Souza e Almeida } \\
\qquad(2013) \\
\text { Artigo } \\
\text { Sócio-histórica: } \\
\text { Vigotsky }\end{array}$ & $\begin{array}{c}10 \\
7 \mathrm{M} 3 \mathrm{~F} \\
12 \text { a } 20\end{array}$ & Artigo & $\begin{array}{l}\text { Foram feitas entrevistas semiestruturadas a fim de se investigar as } \\
\text { concepções de jovens com Deficiência Intelectual, em regime de } \\
\text { escolarização especial, e de seus pais e ou mães, sobre os motivos da } \\
\text { não inclusão escolar dos estudantes. Os dados foram gravados em } \\
\text { áudio. }\end{array}$ & $\begin{array}{l}\text { Dados transcritos e lidos. A partir dos dados foram estabelecidas categorias } \\
\text { temáticas por meio da análise de conteúdo e elaboração de núcleos de } \\
\text { significação e sentidos. Os resultados apontaram para a falta de } \\
\text { empoderamento dos alunos em relação ao seu processo de escolarização e } \\
\text { para a necessidade de se buscar alternativas para a inclusão escolar de } \\
\text { alunos com DI com idades mais avançadas e não alfabetizados e o } \\
\text { redirecionamento das práticas pedagógicas oferecidas pelas unidades de } \\
\text { ensino especial. }\end{array}$ \\
\hline 18 & $\begin{array}{l}\text { Tomaino } \\
\text { (2009) Dissertação } \\
\text { Bioecológica de } \\
\text { Bronfenbrenner }\end{array}$ & $\begin{array}{c}5 \\
3 F 2 M \\
15 \text { a } 26\end{array}$ & Entrevista & $\begin{array}{l}\text { Entrevista semiestruturada a fim de observar a caracterização dos } \\
\text { sujeitos, situação escolar e expectativas futuras. }\end{array}$ & $\begin{array}{l}\text { Levantamento de dados recorrentes e categorização das temáticas a partir } \\
\text { dos objetivos da pesquisa. Definição de classes de categorias de análise a } \\
\text { partir dos temas identificados. Destaque aos tópicos sobre escolarização e } \\
\text { individualidade dos sujeitos. Busca por similaridades e dessemelhanças nos } \\
\text { relatos e organização dos dados em textos que demonstrem as concepções } \\
\text { e percepções dos participantes. Os dados demonstraram ausência de } \\
\text { propostas pedagógicas e organizacionais para garantir o acesso, } \\
\text { permanência e aprendizagem de pessoas com DI na escola regular. }\end{array}$ \\
\hline 19 & $\begin{array}{l}\text { Veltrone e Mendes } \\
\qquad \begin{array}{l}(2009) \\
\text { Artigo }\end{array}\end{array}$ & $\begin{array}{l}10 \\
6 \mathrm{~F} 4 \mathrm{M} \\
10 \text { a } 17\end{array}$ & $\begin{array}{l}\text { Entrevista } \\
\text { estruturada }\end{array}$ & $\begin{array}{l}\text { Participação da aula dos alunos para estabelecer vínculo com os } \\
\text { participantes. Entrevista semiestruturada sobre percepção dos alunos } \\
\text { acerca da aprendizagem em sala de aula regular. }\end{array}$ & $\begin{array}{l}\text { Análise de conteúdo por temas pré-definidos no roteiro de entrevista, } \\
\text { interpretados pela literatura referendada. Dados apontam para dificuldade de } \\
\text { aprendizagem por ausência de adaptação curricular, apesar de os } \\
\text { participantes gostarem da escola. }\end{array}$ \\
\hline 20 & $\begin{array}{l}\text { Veltrone e Mendes, } \\
\text { (2011) } \\
\text { Artigo }\end{array}$ & $\begin{array}{c}10 \\
6 \mathrm{~F} 4 \mathrm{M} \\
10 \text { a } 17\end{array}$ & $\begin{array}{l}\text { Entrevista } \\
\text { estruturada } \\
\text { com produção } \\
\text { temática de } \\
\text { desenhos }\end{array}$ & $\begin{array}{l}\text { Participação da aula pra estabelecer vínculos com os participantes; } \\
\text { Entrevista semiestruturada e produção temática de desenhos } \\
\text { acompanhados de interrogativas sobre percepções de alunos acerca } \\
\text { da escolarização na classe comum e especial e a escolarização de } \\
\text { preferência. }\end{array}$ & $\begin{array}{l}\text { Categorização dos conteúdos das entrevistas e dos desenhos a partir dos } \\
\text { significados expressas pelos alunos e a literatura. Análise de conteúdo e dos } \\
\text { desenhos a partir de temas definidos na pesquisa, com resultados que } \\
\text { apontam para sentimentos positivos ou negativos sobre ambos os sistemas } \\
\text { educacionais, mas com uma preferência pela educação regular. }\end{array}$ \\
\hline
\end{tabular}

